Revue d'histoire de l'Amérique française

BRS REVUE D.HISTOIRE DE L'AMÉRIQUE FRANÇAISE

\title{
Une femme d'affaires en Nouvelle-France : Marie-Anne Barbel, veuve Fornel
}

\section{Lilianne Plamondon}

Volume 31, numéro 2, septembre 1977

URI : https://id.erudit.org/iderudit/303606ar

DOI : https://doi.org/10.7202/303606ar

Aller au sommaire du numéro

Éditeur(s)

Institut d'histoire de l'Amérique française

ISSN

0035-2357 (imprimé)

1492-1383 (numérique)

Découvrir la revue

Citer cet article

Plamondon, L. (1977). Une femme d'affaires en Nouvelle-France : Marie-Anne Barbel, veuve Fornel. Revue d'histoire de l'Amérique française, 31(2), 165-185. https://doi.org/10.7202/303606ar d'utilisation que vous pouvez consulter en ligne.

https://apropos.erudit.org/fr/usagers/politique-dutilisation/ 


\section{UNE FEMME D'AFFAIRES EN NOUVELLE-FRANCE : MARIE-ANNE BARBEL, VEUVE FORNEL}

LiLianNe Plamondon

L'image qu'on retient de la femme du Régime français est celle d'une sainte, d'une héroïne, d'une femme vertueuse ou, tout à l'opposé, de la femme coquette et légère. Et pourtant les Marguerite Bourgeoys, Marie de l'Incarnation, Jeanne Mance, Madeleine de Verchères, Madame de Péan n'ont pas seules marqué la vie coloniale. Certaines femmes se sont lancées dans les activités commerciales et financières à la suite de leur mari ou de leur propre initiative. Rares, très rares, sont les historiens qui se sont attardés à l'étude de ces femmes d'affaires. Parfois mentionnées à l'intérieur d'un article, leurs activités n'ont jamais fait l'objet d'une analyse systématique. Pourtant, la vie de ces femmes dévoile un nouvel aspect de la participation féminine à l'édification de la société coloniale. Ainsi en est-il de Thérèse de Couagne, veuve Poulin de Francheville, Louise de Ramezay, les demoiselles Desaulniers, Agathe de Saint-Père Legardeur de Repentigny et Marie-Anne Barbel, veuve Fornel.

Comme femme active en affaires, Marie-Anne Barbel ne constitue certainement pas une exception pour l'époque. En France, les femmes de marchands bourgeois secondaient assez souvent leur conjoint, et même dans la colonie, des femmes se sont occupées de commerce et d'industrie. Louise Dechêne nous dit d'ailleurs que ce type de femmes existait déjà au XVII ${ }^{\mathrm{e}}$ siècle à Montréal.

Laissées seules, elles se révèlent souvent d'excellentes administratrices, ce qui prouve qu'elles étaient déjà très mêlées à l'entreprise familiale, qu'il s'agisse d'un bien rural ou d'un commerce. ${ }^{1}$

1 Louise Dechêne, Habitants et marchands de Montréal au XVII ${ }^{e}$ siècle (Montréal, Plon, 1974), 439.

RHAF, vol. 31, n $^{\circ}$ (septembre 1977) 
Marie-Anne Barbel appartient à cette catégorie. Toutefois son comportement économique demeure tributaire de son milieu social, du cadre juridique et de la vie commerciale et financière de la Nouvelle-France. La faveur des gens en place dans l'administration coloniale a toujours été une aide précieuse pour les ambitieux; Marie-Anne Barbel l'a appris. Les activités des marchands reposaient sur le commerce des fourrures, et l'exploitation des postes de l'Ouest ou de la côte du Labrador en attirait plusieurs. Si l'obtention de ces postes était grandement facilitée quand le marchand avait de bonnes relations avec l'intendant ou le gouverneur, les relations sociales que se créait un individu avaient aussi une grande importance. Enfin, le cadre juridique imposé par la Coutume de Paris laissait, à la femme célibataire et majeure ou à la veuve, un éventail assez vaste de possibilités.

Il s'agit d'abord de situer le cadre social et économique dans lequel a vécu Marie-Anne Barbel jusqu'à la mort de son mari. Puis l'analyse portera sur la nature, la variété, l'ampleur et le degré de succès des activités auxquelles elle s'est consacrée avant d'évaluei les raisons qui l'ont conduite à se retirer d'une vie commerciale et financière active. Enfin, en examinant les différents aspects de la liquidation de la communauté, il sera possible de saisir la richesse des Fornel que Marie-Anne Barbel a contribué à établir pendant les quinze à vingt années de sa vie de femme d'affaires.

\section{L'environnement social et économique}

Âgée de vingt ans, Marie-Anne Barbel épouse Jean-Louis Fornel en décembre 1723. Cette union rapproche deux familles dont les activités représentent deux secteurs importants de la vie coloniale, l'administration et le commerce. En 1723, Jacques Barbel cumule les fonctions de juge sénéchal de Lauson, notaire royal à Québec, juge bailli de la seigneurie de Beaupré et greffier de l'Officialité $^{2}$. Il est bien connu des dirigeants du pays. Son futur gendre, Jean-Louis Fornel, se qualifie, tout comme son père le faisait, de marchand bourgeois.

Les invités présents au mariage le 31 décembre 1723 donnent l'image d'un réseau de relations sociales assez prestigieux. On y

2 A. Vachon, «Jacques Barbel», Dictionnaire biographique du Canada, tome II (Québec, P.U.L., 1969), 44-45. 
retrouve les plus hauts personnages du pays, le gouverneur, le marquis de Vaudreuil, et l'intendant Bégon et son épouse. S'y joignent Eustache Lanouillier de Boisclerc, contrôleur de la Marine et son épouse, cousine du marié, François Clairambault Daigremont, commissaire de la Marine, Nicolas Gaspard Boucault, secrétaire de l'intendant, et François Daine, greffier en chef du Conseil Supérieur. Ces gens distingués s'unissent pour l'occasion aux parents et aux amis des mariés parmi lesquels figurent Louis Gosselin et sa femme, Jean Crespin, Étienne Veron de Grandmesnil, oncle de la mariée, tous trois marchands bourgeois, et Jean Maillou, architecte du roi ${ }^{3}$. Le couple Barbel-Fornel, par ses origines familiales et ses relations sociales, fait partie de ce qu'on peut appeler la bourgeoisie commerçante; il a certains liens avec des administrateurs dont la protection pourrait lui être fort utile.

La situation financière du jeune couple, telle qu'elle transparaît dans le contrat de mariage, le classe parmi les gens assez aisés. Conformément aux dispositions de la Coutume de Paris, le couple s'unit sous le régime de la communauté de biens. Le mari est le chef suprême de la communauté, c'est lui qui gère les biens. La seule restriction à ce pouvoir total s'applique aux propres de sa femme: il peut «disposer des fruits de la chose comme la récolte d'une terre ou l'intérêt d'une rente, mais non de la chose elle-même sans le consentement de son épouse». Pour cette dernière, c'était là la seule voix qui lui était reconnue dans la direction des affaires familiales. Elle ne pouvait non plus «ester en justice ni se lancer en affaires sans l'autorisation de son mari». ${ }^{4}$ La femme mariée souffre donc d'incapacité juridique presque totale.

Le contrat de mariage de Marie-Anne Barbel et Jean-Louis Fornel comprend toutes les dispositions qu'on retrouve généralement dans ce type d'acte ${ }^{5}$. Des biens qu'il recevra de ses père et mère décédés ${ }^{6}$, Louis Fornel met 2000 livres dans la communauté; le surplus prendra nature de propres. Joachim Fornel, prêtre, donne à

3 ANQ, J. C. Louet père, 31 décembre 1723, Contrat de mariage entre JeanLouis Fornel et Marie-Anne Barbel.

4. Yves Zoltvany, "Esquisse de la Coutume de Paris», RHAF, 25, no 3 (décembre 1971): 369.

5 ANQ, J. C. Louet père, 31 décembre 1723, Contrat de mariage entre Jean-Louis Fornel et Marie-Anne Barbel. réglée.

6 La succession de Jean Fornel, père de Louis Fornel, n'est pas encore 
son frère Louis la somme de 3000 livres en espèces sonnantes à la condition que si Louis meurt sans enfants nés ou à naître, il lui sera possible de reprendre cette somme sur les biens de Louis. Marie-Anne Barbel fait entrer dans la communauté 1500 livres tirées des héritages reçus de sa mère Marie-Anne LePicard, d'une donation que lui a faite son oncle Pierre LePicard, prêtre chanoine, et de ce qui lui reviendra de la succession de son père Jacques Barbel, le surplus ayant nature de propres. Louis Fornel dote sa future épouse du douaire coutumier ou de la somme de 4000 livres de douaire préfix au choix de cette dernière. Le préciput sera loyal et réciproque de la somme de 1000 livres en deniers comptants ou en meubles selon la prisée de l'inventaire. En plus, le survivant aura droit à ses habits, hardes et jupes et à la chambre garnie dans l'état où elle sera au décès du premier mourant. Enfin, les dernières clauses du contrat donnent droit à Marie-Anne Barbel d'accepter ou de renoncer à la communauté et en cas de renonciation, d'emporter ce qu'elle justifiera avoir apporté de même que les biens reçus par succession avec ses douaire et préciput. Elle ne sera tenue d'aucune dette ni hypothèque faites et créées pendant la communauté.

La vie commune du couple Barbel-Fornel dure jusqu'en 1745, année de la mort de Louis Fornel. Des quatorze enfants nés entre 1724 et 1741, neuf meurent en bas âge. Quatre filles et un garçon atteignent l'âge adulte ${ }^{7}$. Deux filles seulement se marient à des commerçants, bien après la mort de leur père. Pendant ces 22 années, Fornel s'occupe de commerce et semble assez bien réussir d'après les investissements qu'il fait. Deux types d'homme d'affaires se succèdent chez Louis Fornel entre 1723 et 1745, correspondant à deux périodes d'activités. Fornel fait figure de marchand détaillant de 1723 à 1737 alors que le commerçant, l'entrepreneur et l'explorateur s'affirment dans les années 1737-1745. Son magasin à Québec bien installé, le marchand se laisse attirer par le commerce des fourrures et, en 1737, devient entrepreneur. Une société regroupe Louis Bazil, Louis Fornel et François Havy dans le but d'exploiter le privilège "de pêche, chasse et traite et de faire des établissements pour la pêche sédentaire du loup-marin à la baye des Chasteaux ${ }^{8}$.

7 C. Tanguay, Dictionnaire généalogique des familles canadiennes depuis la fondation de la colonie jusqu'à nos jours (Montréal, Eusèbe Sénécal et fils, 1887), IV : 84 .

8 ANQ, C. Barolet, 3 mai 1737, Société entre le Sr François Havy, Louis Fornel et Louis Bazil pour la pêche aux loups-marins. 
Havy et Fornel sont les piliers de l'entreprise; le seul apport du marchand Bazil et de son épouse est le privilège d'exploitation qu'ils avaient reçu. Devant le peu d'intérêt que manifeste Bazil pour ce poste, Louis Fornel écrit à Beauharnois et Hocquart en 1742 pour en obtenir la concession.

Dans sa requête il souligne que s'il obtient le poste de la baie des Châteaux, il entreprendra, à ses frais, la découverte de la baie des Esquimaux en se servant de la baie des Châteaux comme point de départ ${ }^{9}$. Sans concéder le poste, Beauharnois et Hocquart accordent à l'entrepreneur la permission de découvrir la baie des Esquimaux. Grâce en partie à l'appui financier de Havy et de Lefebvre ${ }^{10}$, Fornel s'embarque au printemps 1743 laissant la gestion totale et entière de ses affaires à Québec à son épouse ${ }^{11}$. Par cette procuration, Marie-Anne Barbel jouit des mêmes droits que Fornel; elle peut poser tous les actes financiers et juridiques que réclameront les affaires.

Dans les dernières années de sa vie, Fornel oriente donc ses activités avant tout du côté de l'exploitation de postes sur la côte du Labrador. La maladie et la mort l'empêcheront de poursuivre ses projets; sa veuve prendra la relève.

Même si Fornel se laisse tenter par l'aventure en tant que découvreur, il demeure un homme d'affaires prudent qui investit son argent, ou du moins une partie de ses profits, dans le secteur immobilier, moins aléatoire que le commerce.

Entre 1733 et 1745 , Louis Fornel acquiert plusieurs maisons à Québec et des terres aux alentours, soit par héritage ou donation, soit par achat, concession ou adjudication. Il hérite de la maison de son père située à la Place Royale à Québec. Vers 1735, il y fera construire des voûtes ${ }^{12}$. En 1731, il achète un terrain sur le bord de

9 ANQ, Fonds Fornel, Lettre de Louis Fornel à MM. Beauharnois et Hocquart pour obtenir la concession de la baie des Châteaux pendant neuf ans, Québec, 1742.

10 «Apparently, from the time of their arrival in Canada as Dugard and Company's permanent representatives, Havy and Lefebvre were to be reckoned as part of that inner circle of resident metropolitans who dominated the colony's importexport trade.« D. Miquelon, Robert Dugard and the Société du Canada of Rouen, 1729-1770, thèse (University of Toronto, c. 1973), 171.

11 ANQ, C. H. Dulaurent, 15 mai 1743, Procuration par le Sr Louis Fornel à Marie-Anne Barbel, son épouse.

12 Michel Gaumond, La maison Fornel, Place Royale, Québec (Québec, Ministère des Affaires culturelles, 1965), 25. 
la grève au prix de 2100 livres $^{13}$, puis, en avril 1733, un emplacement de trente pieds de front rue Saint-Louis ${ }^{14}$. Par une donation de son frère Joachim en 1734, il devient propriétaire de deux maisons, l'une sur la rue Sous-le-Fort, l'autre située rue du Saultau-Matelot et qu'il louera par la suite ${ }^{15}$. En mai 1741, Beauharnois et Hocquart lui concèdent une seigneurie derrière la seigneurie de Neuville ${ }^{16}$. Puis en 1742, Fornel engage de multiples démarches pour l'achat de la maison voisine de la sienne, Place Royale; sa veuve complétera les transactions après sa mort. Le dernier bien immobilier que Fornel ajoute aux autres lui vient de l'adjudication de la terre de Jean-Baptiste Larchevêque Grandpré appelée «La Briqueterie» au prix de 12000 livres ${ }^{17}$.

La liste des biens immobiliers possédés par Louis Fornel est assez impressionnante: cinq maisons et emplacements dans la ville de Québec dont deux sur la Place Royale; un emplacement rue Saint-Louis, une seigneurie et enfin une terre et ses bâtiments dans la seigneurie Notre-Dame des Anges. En tenant compte du prix d'achat de ces différentes possessions, on peut avancer le chiffre de 40000 livres pour fixer approximativement la valeur monétaire de l'ensemble de ses biens immobiliers.

Jean-Louis Fornel meurt le 30 mai 1745 après une maladie de deux ans. Sa veuve lui donne une sépulture digne de son rang social et dont les frais s'élèvent à 300 livres ${ }^{18}$. Marie-Anne Barbel se retrouve, à 41 ans, seule avec cinq enfants mineurs dont l'aîné a dix-neuf ans et la benjamine six ans. Depuis quelques années, elle a agi à plusieurs reprises comme fondé de pouvoir de son mari; elle apparaît donc initiée aux affaires. Les biens que laisse Jean-Louis Fornel ${ }^{19}$, sa situation familiale et son propre tempérament, influen-

13 ANQ, C. H. Dulaurent, 1er mai 1740. Concession et vente par les dames de l'Hôtel Dieu aud. Louis Fornel.

${ }_{14}$ ANQ, J. C. Louet père, 19 avril 1733, Vente par Jacques Barbel à Louis Fornel et son épouse. Louis Fornel.

15 ANQ, J. C. Louet père, 14 mai 1734, Donation de Joachim Fornel à

16 ANQ, NF-4, Registre d'Intendance, no 9, fol. 8, 14 mai 1741.

17 ANQ, NF-25, Collection de pièces judiciaires et notariales, no 1426, Louis Fornel vs Jean Larchevêque.

18 ANQ, A. J. Saillant, 28 septembre 1771, Compte rendu de Madame Ve Fornel à ses enfants. Fornel.

19 L'auteur n'a trouvé aucune trace d'un testament laissé par Jean-Louis 
ceront la série de choix que s'apprête à faire Marie-Anne Barbel, veuve Fornel.

\section{La femme d'affaires}

La communauté de biens se termine normalement avec la mort de l'un ou l'autre des conjoints. Le partage des biens qui suit à plus ou moins brève échéance laisse la moitié des biens au survivant et divise l'autre moitié entre les héritiers.

Deux possibilités s'offrent à Marie-Anne Barbel: continuer la communauté pendant un certain temps ou procéder au partage avec ses enfants mineurs. Les entreprises de Louis Fornel sont en pleine expansion au moment de sa mort. Sa femme connaît bien ses affaires, elle est encore jeune et en bonne santé; ses enfants mineurs ne peuvent gérer leur part d'héritage et elle devra les entretenir encore plusieurs années. Elle décide donc de continuer la communauté.

Comme veuve, la femme a autant de droits et de pouvoirs que la célibataire. Toutefois, la Coutume de Paris présente toujours le statut de la femme en fonction du mariage. Elle appréhende donc indirectement les droits de la femme célibataire ou veuve. «La femme etant «sui juris» et maitresse de ses biens, de ses droits et de ses actions, elle tombe par le mariage dans la puissance et sous l'autorité de son mari, (...).» ${ }^{20}$ Dans le cas de la femme séparée, «il n'y a aucune raison qui l'empêche de disposer de ses biens à sa volonté, comme si en effet son mari étoit décédé ${ }^{21}$.

En tant que veuve, Marie-Anne Barbel possède donc un statut civil qui ne lui impose aucune restriction si elle veut continuer de faire fructifier les affaires laissées par son mari. Elle prend prudemment la relève dans les premières années qui suivent. Cependant, son esprit d'initiative la conduira à se lancer dans des entreprises de plus grande envergure tout en continuant d'investir une partie de ses profits dans les biens immobiliers.

Au début, l'action de la veuve Fornel s'inscrit dans la continuité des entreprises de son mari. Elle s'occupe du magasin de la Place Royale et décide de continuer la société créée par son mari,

20 C. Ferrière et S. D'Aramond, Commentaires sur la Coutume de la Prévôté et vicomté de Paris (Paris, Les Libraires Associés, 1788), I: 105.

21 Ibid. : 57. 
Louis Bazil et le commerçant François Havy pour le poste de pêche de la baie des Châteaux. De 1745 à 1748 , ce poste ne leur occasionne que des dépenses, surtout à cause de la présence de corsaires anglais dans le golfe. Alors qu'en 1748 l'état normal des choses semble rétabli, la veuve Fornel et ses associés apprennent la concession de la baie des Châteaux à Gaultier, médecin du roi, sous l'allégation qu'ils en avaient abandonné l'exploitation. Ils rédigent une protestation au ministre dans laquelle ils demandent la concession du poste pour six ans, et en cas de refus, un remboursement de 30000 livres pour les pertes encourues par les installations, chaloupes et agrès qu'ils y ont laissés ${ }^{22}$. Mais le médecin demeurera concessionnaire.

Cependant, l'intendant Bigot demande au ministre de concéder à la veuve Fornel le poste de la baie des Esquimaux découvert par Jean-Louis Fornel. Il souligne de plus l'intérêt des commerçants Havy et Lefebvre dans les établissements que pourra y faire la veuve Fornel $^{23}$. Au printemps 1749, le Président du Conseil de la Marine donne carte blanche à Bigot et à La Jonquière. Ils pourront le concéder à la veùive Forninel poúr une période de douze ans, ce qui est fait à l'été ${ }^{24}$. En 1742, Louis Fornel avait déjà demandé cette concession. Le directeur du Domaine d'Occident avait avancé qu'elle pourrait nuire à la ferme de Tadoussac. Et à l'époque, l'oreille de l'intendant Hocquart et celle du ministre en France étaient plus attentives aux propos d'un François-Étienne Cugnet qu'aux représentations du marchand. Fornel n'avait rien obtenu. L'affaire a été oubliée pendant quelques années, jusqu'au moment où, à son tour, Marie-Anne Barbel en fait la demande. La Galissonnière et Bigot considèrent qu'un établissement à cet endroit sera plus rentable s'il est entrepris par un particulier plutôt que pour le compte du roi et qu'à la fin de la période de concession le roi pourra le réunir à la ferme de Tadoussac.

À la même époque, Marie-Anne Barbel offre aussi 7000 livres pour la ferme de Tadoussac. L'intendant Bigot, qui ne manifeste aucune sympathie pour le directeur du domaine et le soupçonne

22 A. Col., Série C 11 A, vol. 92, fol. 187-191, 10 novembre 1748, Representations respectueuses de la Dlle fornel et les Srs havy et lefebvre à Monsieur le comte de Maurepas.

${ }^{23}$ A. Col., Série C 11 A, vol. 92, fol. 229, 8 novembre 1748, Bigot au ministre. tre.

24 A. Col., Série C 11 A, vol. 93, fol. 241, 25 septembre 1749, Bigot au minis- 
d'avoir retiré des profits considérables de la ferme, s'empresse de la céder à la veuve Fornel ${ }^{25}$.

L'intendant Bigot, le contrôleur de la marine Bréard et la veuve Fornel passent le bail de la ferme de Tadoussac le 9 septembre 1749 devant le notaire Lanouillier. Le contrat lie la veuve Fornel pour une période de six ans, du $1^{\text {er }}$ octobre 1749 au $1^{\text {er }}$ octobre 1755 , au prix de 7000 livres par année et comprend différentes charges, clauses et conditions. La veuve Fornel se chargera des prêts faits aux Sauvages dans les postes, des maisons, ustensiles, meubles et bestiaux qui s'y trouvent et des réparations nécessaires. Elle devra rembourser à François-Étienne Cugnet le prix des marchandises qui s'y trouveront et des derniers envois qu'il aura faits. Les castors, huiles, pelleteries et autres effets provenant du poste seront affectés en premier lieu et de préférence au paiement des 7000 livres de la ferme. Le prochain fermier lui rendra le montant des investissements qu'elle aura faits pour 1756. Tous les castors obtenus des traites devront être portés au bureau de la compagnie de castor de Québec. Enfin, la veuve Fornel s'engage à conserver et à augmenter les traites de Tadoussac et à les régir le plus avantageusement possible, sans quoi on lui retirera l'exploitation de la ferme ${ }^{26}$.

Le roi semonce Bigot d'avoir accordé le bail de Tadoussac à la veuve Fornel et lui ordonne de mettre la ferme aux enchères. Bigot s'en tire habilement et la veuve Fornel conservera son bail jusqu'en 1755. Elle administre la ferme avec Havy et Lefebvre, c'est pourquoi elle signe "veuve Fornel et compagnie».

Les frais d'exploitation d'une année régulière comprennent la valeur des effets et marchandises non vendus restés dans les magasins des postes, les envois à faire de Québec, les gages et le fret des bâtiments, et enfin le prix de la ferme. Les sommes investies au cours de la première année s'élèvent à 125766 livres. Quant aux recettes, elles reposent sur les huiles et les fourrures: peaux de castor, loups-cerviers, renards, martres, visons et autres et totalisent un montant de 50428 livres. Le déficit est élevé, mais il faut considérer le fait qu'une première année d'exploitation entraîne des dépenses particulières: frais de voyage des nouveaux fermiers pour assister aux inventaires faits par le commissaire envoyé par l'intendant, rem-

25 Ibid.

26 ANQ, Fonds veuve Fornel, Bail de la ferme de Tadoussac à la Vve Fornel, 9 septembre 1749 . 
boursement à l'ancien fermier de la valeur des meubles, ustensiles, bestiaux qu'il y a laissés et des envois déjà faits, cadeaux aux Saúvages pour se gagner leur faveur ${ }^{27}$.

Le bail de la veuve Fornel expire le $1^{\text {er }}$ octobre 1755 . Le notaire Dulaurent reçoit une commission en vue de procéder à l'inventaire des postes de Tadoussac ${ }^{28}$. Le roi en reprend la régie le 7 octobre 1755. Les anciens fermiers ont apporté des améliorations aux différents postes; le conseiller Guillimin les avait évalués à 44686 livres en 1750; en 1755, les inventaires indiquent une valeur de 71069 livres. Le roi s'engage à rembourser 26383 livres à la veuve Fornel ${ }^{29}$.

Aucun document ne vient jeter une lumière sur la gestion de la ferme de 1751 à 1755 . Toutefois, une estimation de ce que produit le castor vendu chaque année des postes de Tadoussac, Chicoutimi, Islets Jérémie, Rivière Moisy et Sept-Iles situe la recette moyenne entre 18 et 20000 livres. En 1755, la recette est montée à 20930 livres ${ }^{30}$. De plus, par les investissements immobiliers effectués par Marie-Anne Barbel en 1753 (adjudication d'une maison et d'un emplacement au prix de 8000 livres; achat pour la somme de 1300 livres de la part de sa sœur Marguerite dans leur terre de SainteAnne-de-Beaupré), on peut supposer que les fermiers réussissaient à tirer de Tadoussac des profits intéressants.

Marchande, fermière de Tadoussac, la veuve Fornel ajoute un autre volet à ses activités en installant vers 1746 , une poterie sur la terre de «La Briqueterie» ${ }^{31}$. Mais Marie-Anne Barbel rencontre de nombreuses difficultés avec les différents potiers qu'elle engage, ce qui rend la production irrégulière. Après quelques procès avec les potiers, elle abandonne cette activité vers 1752 .

Marie-Anne Barbel se taille auprès de ses contemporains une réputation de femme d'affaires. À partir de 1746, elle multiplie ses activités, sait se gagner la faveur des gens en place, celle de Bigot en particulier, et conserver ses associés, Havy et Lefebvre, dont les

27 A. Col., Série C 11 A, vol. 96, fol. 104v-108. État et Compte des Traittes de Tadoussac... pendant la présente année 1750 .

28 ANQ, NF-2, Ordonnances des Intendants, vol. 42 , fol. $2 \mathrm{r}-3 \mathrm{v}, 18$ juillet 1755.

29 Ibid., fol. 9-10, 28 décembre 1755.

30 A. Col., Série C 11 A, vol. 100, fol. 338 v, «Memoire sur l'Exploitation des Traittes..., Reflexion sur lad.e Exploitation», 19 novembre 1755. à Ve Fornel.

${ }_{31}$ ANQ, C. H. Dulaurent, 6 novembre 1747, Engagement de Michel Choret 
affaires reposent sur une base solide. Elle imprime sa marque personnelle à l'héritage laissé par son mari et en élargit même l'aire d'action.

En tant que marchande et gestionnaire des fruits de propriétés communes avec sa sœur Marguerite, Marie-Anne Barbel eut souvent à défendre ses droits et à réclamer ses biens devant les tribunaux. Mais le procès le plus long (1745-1756) engagé par la veuve Fornel porte sur une contestation entre elle, censitaire, et les pères Jésuites, seigneurs, au sujet de la terre de la Briqueterie située dans la seigneurie de Notre-Dame-des-Anges. Elle y défend l'intégrité de l'un de ses biens immobiliers.

Le litige porte sur dix-neuf arpents cinq perches qui ont été rongés par la rivière Saint-Charles et qui rapetissent ainsi l'étendue de la terre. Marie-Anne Barbel exige que les seigneurs lui restituent les dix-neuf arpents cinq perches ou qu'ils lui versent 6000 livres de dommages et intérêts. Les Jésuites refusent l'une et l'autre des demandes, invoquant le fait, qu'en avril 1689, ils avaient accordé aux anciens propriétaires une augmentation de terre d'environ cinquantedeux arpents en superficie pour suppléer à un enfoncement qu'avait fait la rivière Saint-Charles ${ }^{32}$. Le procès dure depuis huit ans et se promène de la prévôté au Conseil supérieur et à la prévôté. Quatre arpentages ont été effectués. Finalement, les parties en viennent à une entente à l'amiable devant un notaire. Le 9 août 1756, devant maître Dulaurent, les Jésuites et Marie-Anne Barbel signent un acte d'accord final ${ }^{33}$. Les Jésuites abandonnent purement et simplement à madame Fornel tout le terrain qu'elle possède à ce moment. Cette dernière verse 50 livres 17 sols 6 deniers pour solde de dix années d'arrérages de droits seigneuriaux.

La terre de la Briqueterie est l'un des biens immobiliers laissés par Louis Fornel au moment de sa mort. La veuve choisit de poursuivre ce type d'investissement et cette attitude révèle une femme à la recherche d'une sécurité basée sur la diversification de ses avoirs. Elle acquiert, en mars 1746, une terre en bois debout de deux arpents de front sur vingt de profondeur à Charlesbourg au prix de 60 livres payées comptant ${ }^{34}$ et, en novembre 1747 , une terre en concession de

32 ANQ, NF-20, Documents de la Prévôté de Québec, dossier no 1445.

33 ANQ, C. H. Dulaurent, 9 août 1756, Acte d'accord et transaction avec solde de tous comptes entre la Dlle Ve Fornel et les R. P. Jésuites.

34 ANQ, C. H. Dulaurent, 29 mars 1746, Vente par Jacques Loiselle à M. A. Barbel, veuve Louis Fornel. 
trois arpents de front sur trente de profondeur dans la seigneurie de Neuville ${ }^{35}$. Entre 1747 et 1754 , elle complète l'achat des propriétés situées à côté de la sienne sur la Place Royale et appartenant aux héritiers de dame Jeanne Gourdeau veuve Macart. Dans le but de faire construire une nouvelle maison sur cet emplacement voisin et de rembourser une dette, Marie-Anne Barbel emprunte 8000 livres du négociant Charles Berthelot en s'engageant à lui payer 400 livres de rente par année ${ }^{36}$.

En mars 1753, lors d'une vente à l'enchère, un emplacement et une maison situés rue du Cul-de-Sac et Champlain de trente-et-un pieds de front sur trente-deux de profondeur sont adjugés à la veuve Fornel pour 8000 livres $^{37}$. Elle possède une autre maison rue du Cul-de-Sac et de Meule ${ }^{38}$. Enfin, elle loue le terrain et la maison rue Saint-Louis au prix de 70 livres par an ${ }^{39}$. Toutes ces possessions immobilières lui procurent des revenus intéressants.

Elle s'occupe de gérer soigneusement les biens de la communauté sans négliger pour autant ses biens propres. Elle partage les fruits de l'exploitation de deux terres avec sa sœur Marguerite. Ces terres sont situées l'une à Sainte-Anne-de-Beaupré, l'autre à SaintJoachim. Elles leur ont été léguées par leur mère; Marie-Anne est responsable de la gestion de l'ensemble. En mai 1753, elle achète la part de sa sœur dans la terre de Sainte-Anne pour la somme de 1600 livres. Elle jouira désormais seule des profits tirés de cette ferme ${ }^{40}$.

Ces nombreux biens immobiliers ruraux et urbains présentent un autre aspect des investissements monétaires de la veuve face aux fluctuations de l'économie. On retrouve ce type d'investissement chez plusieurs négociants, dont les associés de madame Fornel: Havy et Lefebvre: "Much of their capital was invested in houses and

35 ANQ, C. H. Dulaurent, 6 novembre 1747, Vente par André Campagna à M. A. Barbel.

36 ANQ, C. H. Dulaurent, 15 octobre 1750, Constitution de 400 livres de rente au principal de 8000 par Ve Fornel à Charles Berthelot. 20 mars 1753.

37 ANQ, NF-19, Registres de la Prévôté de Québec, vol. 96, fol. 46r et v,

38 Nous n'avons retracé aucun document concernant l'acquisition de cette maison. Son existence nous est connue par mention dans un autre document: J. B. Decharnay, 7 avril 1758, Bail de la Ve Fornel à la Ve de Jacques Marseron.

39 ANQ, NF-25, CPJN, no 1586, Dlle Ve Fornel vs Blaise Perrot.

40 ANQ, J. C. Panet, 4 mai 1753, Vente de Pierre Costé et son épouse à M. A. Barbel veuve Fornel. 
properties in and around Quebec and in mortgages providing them with some immunity against the fickle ups and downs of trade ${ }^{41}$.»

Par ses activités, Marie-Anne Barbel s'est taillé une place parmi les marchands reconnus. On est porté à croire que sa situation a favorisé des mariages avantageux pour ses enfants. La première des demoiselles Fornel qui se marie est la benjamine, Louise, qui épouse en janvier 1758, un jeune marchand, Charles Parent, lui-même fils de marchand. Cette union est scellée à un moment peu favorable aux affaires de madame Fornel. Dans le contrat de mariage, la mère de la mariée promet de faire l'inventaire des biens de la communauté qui a été entre elle et son mari aussitôt après l'arrivée des vaisseaux de France $^{42}$. Or l'inventaire sera dressé en 1765 , après la séparation de Louise et Charles. Les invités au mariage se concentrent surtout chez les marchands, Jean Lefebvre, les frères Tropez et Barthelemy Martin, Jean Taché, Barthelemy Cotton; quelques conseillers du Conseil supérieur sont aussi présents dont Thomas Cugnet. Les invités à ce mariage sont presque tous issus de la même classe. La famille de la veuve Fornel semble bien intégrée à cette classe de marchands. Françoise épouse Antoine-Florent Meignot au début des années 1760 et, quelques années après la mort de celui-ci, elle deviendra la femme d'Alexandre Dumas. Les deux autres filles et Jean-Louis, fils unique, demeureront célibataires.

$\mathrm{Au}$ cours de toutes ces années, Marie-Anne Barbel continue donc la communauté qui a été entre elle et son mari, ce qui lui permet de se lancer dans différents types d'activités et d'en tirer de multiples sources de revenus. Mais quelques coups viennent ébranler l'édifice. En 1755 , le bail de la ferme de Tadoussac se termine et n'est pas renouvelé. Les difficultés d'entente avec les différents potiers entraînent finalement l'abandon de la poterie. La situation économique et militaire de la colonie n'est pas reluisante, la présence des Anglais dans le golfe ralentit les échanges commerciaux. La guerre hypothèque sérieusement l'avenir de Marie-Anne Barbel.

\section{La liquidation des affaires}

La Conquête vient donner le coup de grâce à plusieurs carrières commerciales et financières dont celle de Marie-Anne Barbel.

41 D. Miquelon, «Havy and Lefebvre of Quebec: A Case Study of Metropolitan Participation in Canadian Trade, 1730-60», CHR, LVI, no 1 (mars 1975): 17.

42 ANQ, C. H. Dulaurent, 14 décembre 1757, Dépôts des articles du mariage de Louise Fornel et Ant. Charles Parent. 
Après le bombardement de Québec qui a endommagé plusieurs de ses maisons dont le magasin de la Place Royale, on ne retrouve plus le nom de Marie-Anne Barbel dans les actes notariés. Elle ne semble pas avoir exercé d'activités commerciales après 1759. La veuve de Louis Fornel se décide enfin à procéder à la liquidation de la communauté qu'elle avait continuée depuis la mort de son mari. Cette opération s'étend sur plusieurs années. Marie-Anne Barbel, malgré son âge avancé, demeure d'esprit alerte et lucide. Elle conserve son sens des affaires et demeure soucieuse de ses droits et de ce qui lui revient de la communauté. Elle ne s'en laisse pas imposer par ses enfants.

En 1765, elle fait dresser l'inventaire de ses biens. À ce moment, trois de ses filles demeurent avec elle: Joachim Charles (ou Charlotte $)^{43}$, Anne Claire, toutes deux célibataires, et Louise, épouse séparée de Charles Parent ; ce dernier a quitté la colonie. Son gendre Antoine-Florent Meignot l'aidera à régler certaines dettes et à récupérer la valeur de différentes lettres de change.

L inventaire des biens est étabil par Ciaude Louet ${ }^{44}$. La iiste des biens immobiliers est longue. Une première maison héritée de Jean Fornel est située sur la Place Royale. La maison voisine de celle-ci est érigée par Marie-Anne Barbel entre 1753 et 1755 . Un terrain de cent trente pieds de front appelé «La Canoterie» s'étire sur le bord de la grève. Les bâtiments qui y étaient construits ont été incendiés en 1759. Un terrain de soixante pieds de front rue SaintLouis à la haute ville leur appartient aussi. À cela, il faut ajouter un emplacement et la maison rue Sous-le-fort de trente pieds de front sur vingt pieds de profondeur donnés par Joachim Fornel, moyennant une pension viagère. Enfin, une maison et son terrain situés rue du Culde-Sac et rue de Meulles font aussi partie des biens.

À ces possessions urbaines s'ajoutent quelques terres à la campagne. Une seigneurie de deux lieues et trois quarts de front sur trois lieues de profondeur derrière la seigneurie de Neuville avait été concédée à Jean-Louis Fornel en 1741. Mais ni le nouveau seigneur, ni son épouse plus tard, ne se sont occupés de la développer. En 1744, Fornel se voyait adjuger une terre et habitation de six arpents de

43 Le cas de Joachim Charles ou Charlotte demeure étrange: pourquoi lui a-t-on donné un nom de garçon sans équivoque à sa naissance? Et pourquoi, plus tard, l'appelle-t-on Charlotte, même si elle signe les actes notariés Joachim Charles?

44 ANQ, Claude Louet fils, 10 mai 1765, Inventaire de biens de Dlle MarieAnne Barbel et S. Louis Fornel. 
front sur quarante de profondeur dans la seigneurie de Notre-Damedes-Anges sur la petite rivière Saint-Charles. On y retrouve une maison de pierres à un étage de soixante pieds par vingt-cinq pieds, une grange avec une étable attenante, une petite écurie, un hangar, un fourneau en briques et une petite maison de trente pieds par vingt pieds. Enfin, une terre en bois debout de deux arpents de front sur vingt arpents de profondeur dans la seigneurie Notre-Dame-desAnges vient compléter la liste des biens immobiliers. Deux autres propriétés auraient dû s'ajouter à cette liste, mais l'emplacement et la maison achetés rue du Cul-de-Sac et Champlain en 1753 ont été vendus en avril 1764 au prix de 5600 livres $^{45}$ et la terre en bois debout située dans la seigneurie de Neuville et achetée en 1747 sera abandonnée au seigneur le ler août $1765^{46}$.

Les biens mobiliers comprenant argenterie, animaux, ustensiles, meubles, vaisselle, linge, argent en lettres de change et en billets et ordonnances du trésor de la marine, sont évalués à 8800 livres dont 1105 livres uniquement pour l'argenterie.

TABLEAU I

Dettes actives et passives ${ }^{47}$

Dettes actives

par les héritiers de feu Mess. Chardon vivant cure de la paroisse de Beauport la somme de

par M. Duplessis faber la somme de suivant son billet du $1^{\text {er }}$ avril 1754 laquelle somme sad. Dame veuve a esté obligé d'accepter en payement de la nommée sanson pour loyer

par le S. Michel Riverin neg. $t$ la somme de

$63615 \mathrm{~s}$

pour fournitures a luy faites de divers effets en 1758 et 1759

Dettes passives

au S. Charles berthelot, neg. $\mathrm{t}$ la somme de pour un Consitut par Elle consenti au

45 ANQ, J. C. Panet, 2 avril 1764, Vente de M. A. Barbel à George Borne.

46 ANQ, S. Sanguinet, ler août 1765, Abandon et déguerpissement de M. A. Barbel à N. M. Renaud d'Avaines Desmeloizes.

47 ANQ, Claude Louet fils, 10 mai 1765, Inventaire Barbel-Fornel. 
profit dudit S. Berthelot suivant la grosse dud. contrat de constitution passé devant Me Dulaurent no. royal

au S. Berthelot la somme de

pour les arrerages de lad. rente depuis 1759 compris jusqu'au $1^{\text {er }}$ octobre prochain

a $\mathrm{Mr}$ françois havy residant a Bordeaux la somme de

pour restant de plus grosse due par lad. communauté suivant la transaction faite avec le S. L'Evesque negociant de cette ville au nom et comme fonde de procuration dud. S. havy lad. transaction passée devant Me Panet

au S. Gaudet negociant de Montreal la somme de

pour argent qu'il a presté a lad. Dame veuve pour le rétablissement des maisons apres l'incendie

aux S. Chartier et Bondfield negociants a Quebec la somme de pour solde de compte suivant leur memoire du 10 du present mois

plus la somme de

pour fourniture de serrure qu'il a faite pour la maison dependant de la communauté suivant son memoire du 18 juin 1763

au S. Jean Baptiste Charpentier negociant a Quebec la somme de

pour argent par lui presté a lad. veuve pour payer les divers ouvriers qui ont travaillé au retablissement des deux maisons sizes sur la place du marché et rue Saint-Pierre suivant les differents reçus des ouvriers.

Il est évident que les livres de comptes de la marchande Fornel ont été mis à jour auparavant et que la presque totalité des dettes dues à la communauté ont été récupérées avant la tenue de l'inventaire. Les dettes passives apparaissent plus importantes; les plus élevées ont été contractées avant la Conquête. La construction de la maison voisine de la leur, Place Royale, s'est faite grâce à l'emprunt effectué auprès de Charles Berthelot. Quant à François Havy, il était 
associé de la veuve Fornel et c'est au cours de leurs années de collaboration que cette dernière s'est endettée de cette façon. Les dettes passives s'élèvent à 24330 livres; la somme est imposante, mais on verra que madame Fornel réussira à tout rembourser. Les Fornel ont été des gens à l'aise avant la Conquête; ils se retrouvent six ans après la défaite française avec les reliquats d'une richesse passée, reliquats qui demeurent toutefois intéressants comme le démontre le partage effectué en 1777.

En juin 1765, on procède à la vente à l'enchère des biens meubles de la communauté Barbel-Fornel ${ }^{48}$. 3985 livres sont retirées de la vente alors que la prisée de l'inventaire avait évalué les mêmes articles à 2095 livres. La liquidation de la communauté se poursuit donc tranquillement pendant que Marie-Anne Barbel se défait de quelques-uns de ses biens propres avec l'autorisation de ses enfants. Elle vend deux terres dont elle partage la possession avec son frère et sa sœur; elles lui rapportent 4250 livres, monnaie de la province ${ }^{49}$. La veuve Fornel continue à garder la main haute sur la gestion des affaires de famille.

En 1771, elle rend compte à ses enfants de sa gestion des biens de la communauté depuis $1765^{50}$. Fait intéressant, elle préfère l'aide d'un gendre averti en affaires, Antoine-Florent Meignot à celle de son fils Jean-Louis âgé de 46 ans.

Le partage des biens de la communauté Barbel-Fornel a lieu trente-deux ans après la mort de Fornel, douze ans après l'inventaire et six ans après le compte rendu de la veuve. Les cas de communautés qui durent trente-deux ans après la mort de l'un des conjoints doivent être plutôt rares et supposent des conditions ou une volonté particulière. Les raisons qui expliquent les douze ans qui séparent l'inventaire du partage sont exprimées dans l'acte de partage luimême: "Après cet inventaire et vente si médiocre les enfans de monsieur Fornel demeurent avec leur mere qui les soutenait de son mieux sans oser lui demander compte et partage parce qu'ils n'ignoraient pas qu'il falloit payer les trente mille livres dues: ils se contenterent donc de vivre de communaute avec elle en se soutenant par

48 ANQ, Claude Louet fils, 10 juin 1765, Procès-verbal de la vente de meubles de Dlle Ve Fornel. Talon.

49 ANQ, Claude Louet fils, 23 août 1765, Vente par M. A. Barbel à Jacques à ses enfants. 
le secours des loyers que produisoient les maisons ${ }^{51} »$. S'ils n'avaient pas attendu toutes ces années, les héritiers Fornel auraient peut-être été obligés de renoncer à la succession, alors qu'après avoir attendu douze ans, ils recevront un héritage assez élevé.

L'acte de partage se divise en trois grandes parties: ce qui concerne la communauté d'abord, les propres ensuite et les dépenses communes de la veuve et des trois enfants demeurés avec elle. Les biens de la communauté sont évalués à 72370 livres alors que les dettes montent à 39209 livres. Les biens immobiliers représentent $57 \%$ de la somme totale des avoirs.

Avant de procéder au partage il reste 22252 livres à la communauté. Cette masse liquide est divisée moitié à la veuve, moitié aux enfants. Mais avant de subdiviser cette somme entre les enfants, il faut déduire la moitié des frais funéraires et du deuil occasionnés par la mort de Louis Fornel et la moitié du prix de la seigneurie à cause du droit d'aînesse de Louis ${ }^{52}$; il reste donc 10176 livres à diviser entre les cinq enfants. Louis Fornel fils reçoit 2335 livres pour son droit d'aînesse et sa part de la communauté, ses sœurs 2100 livres pour leurs parts du fief et de la communauté.

\begin{tabular}{|c|c|c|c|}
\hline \multicolumn{4}{|c|}{$\begin{array}{c}\text { TABLEAU II } \\
\text { Récapitulation de la part de chaque enfant }\end{array}$} \\
\hline enfant & description des biens & sous-total & total \\
\hline Louis & $\begin{array}{l}\text { droit d'aînesse sur fief de Bourg } \\
\text { Louis } \\
\text { part des biens de la communauté } \\
\text { le } 1 / 5 \text { des propres fictifs } \\
\text { part du prix de la maison et du } \\
\text { reste des loyers }\end{array}$ & $\begin{array}{ll} & 300 \\
2 & 035 \\
2 & 022 \\
& 3902\end{array}$ & 8259 \\
\hline $\begin{array}{l}\text { chacune des } \\
\text { quatre filles }\end{array}$ & $\begin{array}{l}\text { part du fief de Bourg Louis } \\
\text { part des biens de la communauté } \\
\text { le } 1 / 5 \text { des propres fictifs } \\
\text { part du prix de la maison et du } \\
\text { reste des loyers }\end{array}$ & $\begin{array}{r}65 \\
2035 \\
2022 \\
3.902\end{array}$ & 8024 \\
\hline
\end{tabular}

51 ANQ, J. A. Panet, 21-24 octobre 1777, Compte et partage des biens de feu Sr Louis Fornel.

52 Le droit d'aînesse ne s'applique que sur les biens dits nobles, donc sur la seigneurie. Voir Y. Zoltvany, loc. cit., 378. 
On procède ensuite au partage des propres. Marie-Anne Barbel retient pour la rente du douaire coutumier la moitié des loyers de la maison qui est le propre de son mari, soit 2451 livres; la même somme échoit aux cinq enfants. La part de chaque enfant dans les propres fictifs monte à 2022 livres et le prix entier de la maison et le reste des loyers donnent à chacun 3902 livres. Il revient donc à chacune des quatre filles 8024 livres et à Louis, à cause de son droit d'aînesse, 8259 livres.

Un compte particulier est dressé pour les trois enfants qui vivaient avec la veuve Fornel (Louis, Anne-Claire et Joachim). Chacun remboursera 1310 livres à sa mère.

Le principe d'égalité entre les cinq enfants est respecté et la part qui revient à chacun diffère assez peu.

TABLEAU III

Partage final des biens

masse réelle

50130

Louis Fornel

6939

Anne Claire Fornel

6714

Joachim Charles Fornel

6714

Marie-Françoise Fornel, veuve Meignot

8024

Louise Fornel, épouse séparée de Charles Parent

8024

Marie-Anne Barbel, veuve Fornel

L'acquisition de chacun de ces montants se fait par la perception de loyers et dettes dues. Avant le partage, il y a eu adjudication des différents immeubles de la communauté. La veuve Fornel garde la maison rue Sous-le-Fort et s'y installe avec trois de ses enfants. Jean-Louis, Anne-Claire et Joachim Charles acquièrent la maison de la Place Royale. Antoine Panet devient seigneur de Bourg Louis et Alexandre Dumas adjudicataire de la maison du coin voisine de la maison Fornel, Place Royale. C'est en tenant compte aussi de ces adjudications que chacun recouvre sa part. Les clauses du contrat de mariage de 1723 ont été respectées. La veuve a choisi le douaire coutumier dont le capital a été fixé à 4679 livres. 
Pour la veuve Fornel, la période 1777-1793 est celle de la vieillesse tranquille ponctuée de quelques épreuves: la mort de son fils Jean-Louis en 1784 et celle de Anne-Claire en septembre 1793. Quelques jours après la mort de Anne-Claire, Marie-Anne Barbel rédige un testament devant le notaire Planté par lequel elle lègue à Françoise, dame Dumas, 300 livres et à Louise, veuve Parent, 150 livres et institue Joachim Charles légataire universelle de tous ses autres biens meubles et immeubles ${ }^{53}$. Marie-Anne Barbel s'éteint le 16 novembre 1793 à l'âge de 90 ans.

Après la mort de son mari, la veuve Fornel imprime sa marque personnelle à l'orientation des affaires familiales. Elle obtient non seulement la concession du poste de la baie des Esquimaux au printemps 1749, mais aussi le bail de la ferme de Tadoussac qu'elle gérera en compagnie avec Havy et Lefebvre, pendant six ans. Marie-Anne Barbel a réussi là où son mari a échoué, mais les gens en place ont changé depuis 1742 .

Tentant de diversifier ses opérations, la veuve Fornel exploite pendant queiques années une poterie sur la terre de la Briqueterie. Cette affaire se révèle un échec. Cette poterie fournira à Marie-Anne Barbel plus de tracasseries judiciaires que de terrines à installer sur les tablettes de son magasin de la Place Royale.

Les activités de madame Fornel connaissent un ralentissement à partir de 1755, au moment où le bail de la ferme de Tadoussac n'est pas renouvelé et où François Havy retourne en France pour voir à ses affaires là-bas. Cependant, comme elle a su investir aussi dans le secteur immobilier, Marie-Anne Barbel conserve une position financière confortable. Les nombreuses maisons que possède la communauté sont louées et assurent certains revenus et les terres tirées de son héritage propre fournissent des rentes. Mais la guerre et le bombardement de Québec viennent sérieusement ébranler ses affaires. La succession de Louis Fornel (qui est plus celle de l'administration de Marie-Anne Barbel) évaluée à 50130 livres ne situe pas sa veuve parmi les gens d'affaires de grande envergure. Elle ne peut se comparer à un Jacques Perreault l'aîné par exemple ${ }^{54}$ ou aux membres de la «Grande Société».

53 ANQ, P. L. Deschenaux, 7 et 8 février 1794, Compte de succession de Joachim Charles Fornel à ses sœurs.

54 Jacques Mathieu, "Un négociant à Québec à l'époque de la Conquête, Jacques Perreault l'aîné », $R A N Q, 48$ (1970): 29-81. 
À côté des grands brasseurs d'affaires de la colonie, se trouve un grand nombre de marchands plus modestes, mais dont les affaires ont une certaine importance. On peut inclure Marie-Anne Barbel dans ce dernier groupe, car elle a été une femme active en affaires. Marie-Anne Barbel a su conserver des associés très utiles et prendre des initiatives, s'assurer pendant un certain temps une protection indispensable et se placer à la tête de la gestion de la ferme de Tadoussac.

Plusieurs autres femmes ont été aussi actives en affaires que la veuve Fornel. Pourquoi n'étudierait-on pas les activités financières de ces saintes et de ces hérö̈nes traditionnelles, les Marie de l'Incarnation, Jeanne Mance, Marguerite Bourgeoys, Marguerite d'Youville? Elles y gagneraient un visage plus humain et moins mystique et certaines de ces études nous donneraient de plus l'occasion de connaître les ressources et opérations financières des communautés religieuses sous le Régime français. L'image de la femme en NouvelleFrance gagnerait à être renouvelée par une problématique différente, celle qui gravite autour de la femme d'affaires. 